\title{
Nonlinear anisotropy in silicon nanoparticle oligomers
}

M. K. Kroychuk, E. V. Melik-Gaykazyan, A. S. Shorokhov, D.-Y. Choi, V. V. Zubyuk, T. V. Dolgova, M. R. Shcherbakov, D. N. Neshev, A. A. Fedyanin, and Y. S. Kivshar

Citation: AIP Conference Proceedings 1874, 040023 (2017);

View online: https://doi.org/10.1063/1.4998096

View Table of Contents: http://aip.scitation.org/toc/apc/1874/1

Published by the American Institute of Physics

\section{Articles you may be interested in}

Ultrafast modulation of femtosecond laser pulses in direct-gap semiconductor metasurfaces with magnetic resonances

AIP Conference Proceedings 1874, 030044 (2017); 10.1063/1.4998073

Second harmonic splitting in silicon nanoparticles under ultrashot-pulse excitation

AIP Conference Proceedings 1874, 040031 (2017); 10.1063/1.4998104

Nonlinear light scattering by high-index dielectric nanoparticles

AIP Conference Proceedings 1874, 040046 (2017); 10.1063/1.4998119

Electrically tunable all-dielectric optical metasurfaces based on liquid crystals

Applied Physics Letters 110, 071109 (2017); 10.1063/1.4976504

Reconfigurable c-Si/Au hybrid nanoantenna

AIP Conference Proceedings 1874, 030006 (2017); 10.1063/1.4998035

The correlation between magneto-optical response and magnetic dipole resonance excitation in subwavelength silicon-nickel nanogratings

AIP Conference Proceedings 1874, 030026 (2017); 10.1063/1.4998055 


\title{
Nonlinear Anisotropy in Silicon Nanoparticle Oligomers
}

\author{
M. K. Kroychuk ${ }^{1, a)}$, E. V. Melik-Gaykazyan ${ }^{1}$, A. S. Shorokhov ${ }^{1}$, D.-Y. Choi ${ }^{2}$, \\ V. V. Zubyuk ${ }^{1}$, T. V. Dolgova ${ }^{1}$, M. R. Shcherbakov ${ }^{1}$, D. N. Neshev ${ }^{3}$, \\ A. A. Fedyanin ${ }^{1}$ and Y. S. Kivshar ${ }^{3}$ \\ ${ }^{1}$ Lomonosov Moscow State University, Faculty of Physics, 119991, Moscow, Russia \\ ${ }^{2}$ The Australian National University, Laser Physics Centre, Canberra 2601 ACT, Australia \\ ${ }^{3}$ The Australian National University, Nonlinear Physics Centre, Canberra 2601 ACT, Australia \\ a)Corresponding author: kroychuk@nanolab.phys.msu.ru
}

\begin{abstract}
In this work, we demonstrate experimentally and numerically an anisotropic nonlinear optical response from silicon nanoparticle oligomers. By monitoring the third-harmonic generation from symmetric silicon nanodisk trimers as a function of the linear pump polarization orientation, we observe considerable variations in the nonlinear signal stemming from the polarization sensitivity of the local field profiles, which otherwise play no role in the symmetry of the nanostructure linear response.
\end{abstract}

\section{INTRODUCTION}

The effects of symmetry in the interaction of nanostructures with an electromagnetic field [1] have recently attracted much attention due to prospects of creating novel photonic devices [2]. Linear anisotropy can arise from alteration of the nanostructure geometry $[3,4]$. Anisotropy can also be observed in the nonlinear regime, for example, in spatially anisotropic nanostructures. However, in the most nanostructures studied earlier, the anisotropy can affect simultaneously both linear and nonlinear responses. The nonlinear anisotropy with an isotropic linear response has been studied in plasmonic oligomers [5]. Dielectric oligomers are also considered in terms of nonlinear optical applications (for example, [6]). In this work, we demonstrate experimentally and numerically the type of nonlinear symmetry breaking described above in silicon nanoparticle oligomers by monitoring the third-harmonic generation (THG) signal magnitude while changing the orientation of initial linear pump polarization.

\section{METHODS}

The samples under study were fabricated out of a thin film of hydrogenated amorphous silicon with a thickness of $270 \mathrm{~nm}$ grown on a coverslip of fused silica, with subsequent usage of electron beam lithography and reactive ion etching methods. The obtained structure consists of silicon oligomers trimers composed of three equidistantly placed nanodisks (Fig. 1, right); each array contains disks of the specific values of the disk sizes and distances between them. For each trimer array, a corresponding isolated nanodisk array was fabricated (Fig. 1, left).

The first stage of this work consisted of calculating by the finite-difference time domain method (FDTD) the transmittance spectra of oligomers at different input polarization angles, which demonstrated the absence of polarization sensitivity and the dependence of transmittance on polarization of the normally incident radiation. An experimental setup for linear spectroscopy with possibility of rotating pump beam polarization (Fig. 2, a) was constructed, and linear-optical transmittance spectroscopy was conducted for different pump beam polarization. In agreement with numerical calculations, one observes electric and magnetic dipolar (MD) resonances, and it is apparent that the linear response does not depend on the polarizations of the input beam. 

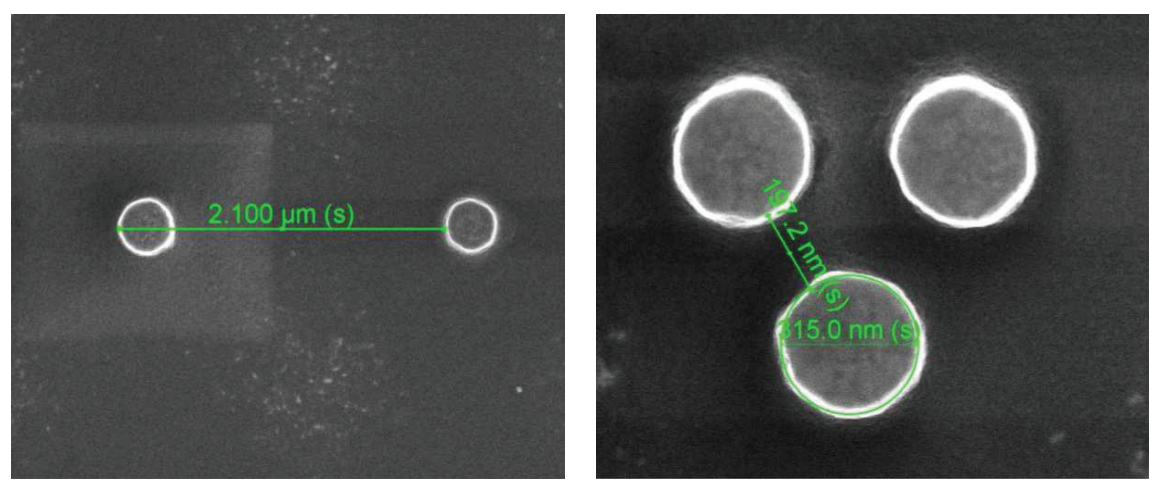

FIGURE 1. Scanning electron micrographs of isolated amorphous silicon nanodisks (left panel) and nanodisk oligomers (right panel).

The next stage of this work consisted of FDTD calculations of local electromagnetic field profiles for arrays of silicon nanodisk trimers for different linear polarization of incident light. We demonstrate the strong electromagnetic field localization for pump wavelengths around the MD resonance. In addition, one observes the dependence of the localization degree and spectral position of the localization maximum on the polarization angle of incident light. The numerical study of THG intensity distribution was carried out. In accordance with the local field analysis, we observe the manifestations of nonlinear anisotropy: THG strongly depends on the polarization of the input beam.

For experimental investigation of the nonlinear anisotropy, an experimental setup based on a femtosecond Ti:sapphire laser and an optical parametric amplifier was constructed (Fig. 2, b). The incident light intensity was controlled by a polarizer placed on a rotating stage, after which a Glan prism was placed. Then we carried out the rotation of linear pump polarization by rotating an achromatic half-wave plate, after which the radiation was focused at the sample. In our experiment, we detected the reflected light at the frequencies that correspond to the third harmonic radiation with a photomultiplier coupled to a boxcar averager. The pump wavelength we chose according to the field localization maximum of the structure. Before conducting the nonlinear measurements, we checked that the detected signal intensity dependence on the incident light corresponded to a cubic one.
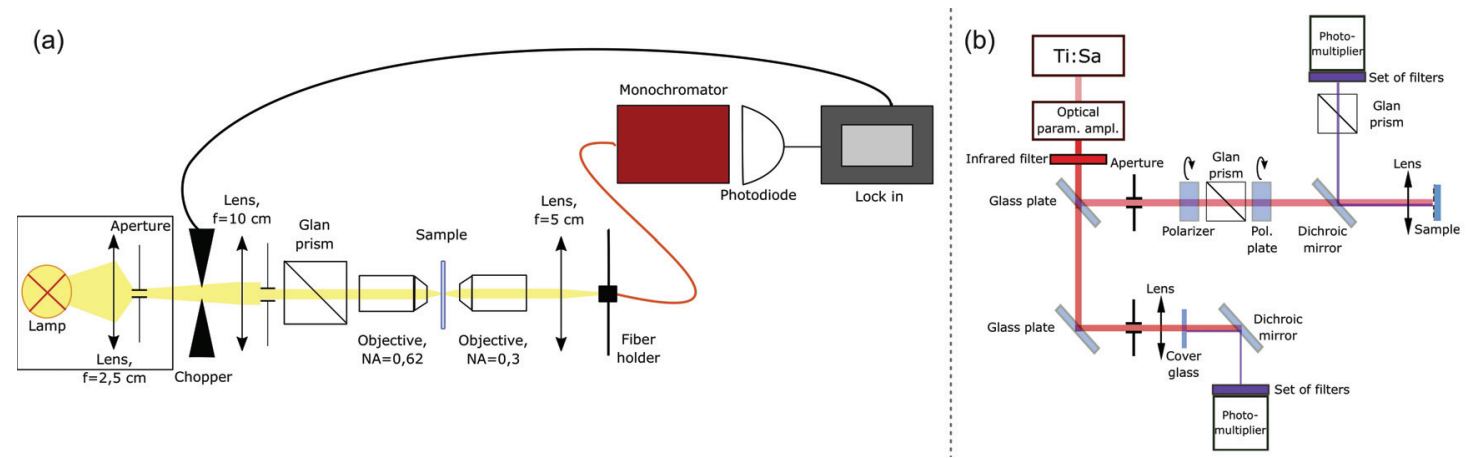

FIGURE 2. Linear (a) and nonlinear (b) spectroscopy experimental setups.

\section{RESULTS AND DISCUSSION}

We obtain the THG intensity dependence on the orientation of the pump polarization for arrays of silicon oligomers and monomers. The main result of this work is the observation of the polarization-insensitivity break in the nonlinear 
optical response around the Mie type MD resonance for trimer samples and the absence of this peculiarity in monomer samples. The linear response is isotropic for both structures. The calculations of the THG and linear spectra done via nonlinear and linear finite-difference time-domain methods are in qualitative agreement with the experimental result. Thereby, in this work we have experimentally and numerically demonstrated the possibility of system anisotropy control while changing its geometry by coupling nanoobjects in oligomers and having, at the same time, an isotropic linear response. Such effects can be applied in symmetry analysis of more complicated nanostructures and molecules.

\section{ACKNOWLEDGMENTS}

The authors acknowledge the support by the Russian Ministry of Education and Science (\#14.W03.31.0008), the Russian Foundation for Basic Research (\#16-29-11811), and the Australian Research Council.

\section{REFERENCES}

[1] N. J. Halas, S. Lal, W.-S. Chang, S. Link, and P. Nordlander, Chem. Rev. 111, 3913-3961 (2011).

[2] M. Durach, A. Rusina, M. I. Stockman, and K. Nelson, Nano Lett. 7, 3145-3149 (2007).

[3] F. Hao, Y. Sonnefraud, P. V. Dorpe, S. A. Maier, N. J. Halas, and P. Nordlander, Nano Lett. 8, 3983-3988 (2008).

[4] F. Hao, P. Nordlander, Y. Sonnefraud, P. V. Dorpe, and S. A. Maier, ACS Nano 3, 643-652 (2009).

[5] M. Rahmani, A. S. Shorokhov, B. Hopkins, A. E. Miroshnichenko, M. R. Shcherbakov, R. Camacho-Morales, A. A. Fedyanin, D. N. Neshev, and Y. S. Kivshar, ACS Photonics 4, 454-461 (2017).

[6] M. R. Shcherbakov, A. S. Shorokhov, D. N. Neshev, B. Hopkins, I. Staude, E. V. Melik-Gaykazyan, A. A. Ezhov, A. E. Miroshnichenko, I. Brener, A. A. Fedyanin, and Y. S. Kivshar, ACS Photonics 2, 578-582 (2015). 